\title{
ON HELE-SHAW MODELS WITH SURFACE TENSION
}

\author{
JoAchim Escher And Gieri Simonett
}

\begin{abstract}
It is shown that surface tension effects on the free boundary have a regularizing effect for Hele-Shaw models, which implies existence and uniqueness of classical solutions for general initial domains.
\end{abstract}

\section{Introduction and main results}

Recently, N. Alikakos, P. Bates, and X. Chen [1] proved that level surfaces of solutions to the Cahn-Hilliard equation tend to solutions of the two-phase Hele-Shaw problem with surface tension under the assumption that classical solutions of the latter exist. In the present note we are able to guarantee that the above assumption is in fact satisfied. More precisely, our results [11] show the existence of a unique classical solution to oneand two-phase Hele-Shaw models with surface tension for general initial data. It should be emphasized that even weak solutions to Hele-Shaw models with surface tension were not known to exist in the general setting presented here. In this note we only give the statements of our results and a brief sketch of their proofs. The full details will appear in [11].

We first consider the one-phase problem. Let $\Omega$ be a bounded domain in $\mathbb{R}^{n}$ and assume that its boundary $\partial \Omega$ is of class $C^{\infty}$. Moreover, assume that $\partial \Omega$ consists of two disjoint non-empty components $J$ and $\Gamma$. Later on, we will model over the exterior component $\Gamma$ a moving interface, whereas the interior component $J$ describes a fixed portion of the boundary. Let $\nu$ denote the outer unit normal field over $\Gamma$ and fix $\alpha \in(0,1)$. Given $a>0$, let

$$
\mathcal{U}:=\left\{\rho \in C^{2+\alpha}(\Gamma) ;\|\rho\|_{C^{1}(\Gamma)}<a\right\} .
$$

For each $\rho \in \mathcal{U}$ define the map

$$
\theta_{\rho}:=i d_{\Gamma}+\rho \nu
$$

and let $\Gamma_{\rho}:=\operatorname{im}\left(\theta_{\rho}\right)$ denote its image. Obviously, $\theta_{\rho}$ is a $C^{2+\alpha}$ diffeomorphism, mapping $\Gamma$ onto $\Gamma_{\rho}$ provided $a>0$ is chosen sufficiently small. In addition, we assume that $a>0$ is small enough such that $\Gamma_{\rho}$ and $J$ are disjoint for each $\rho \in \mathcal{U}$. Let $\Omega_{\rho}$ denote the domain in $\mathbb{R}^{n}$ being diffeomorphic to $\Omega$ and whose boundary is given by $J$ and $\Gamma_{\rho}$. To describe the evolution

Received October 25, 1995. 
of the hypersurface $\Gamma_{\rho}$, fix some $T>0$. Then each map $\rho:[0, T] \rightarrow \mathcal{U}$ defines a collection of hypersurfaces $\Gamma_{\rho(t)}$ and domains $\Omega_{\rho(t)}, t \in[0, T]$. Let us also introduce the following generalized parabolic cylinder

$$
\Omega_{\rho, T}:=\left\{(x, t) \in \mathbb{R}^{n} \times(0, T] ; x \in \Omega_{\rho(t)}\right\}=\bigcup_{t \in(0, T]}\left(\Omega_{\rho(t)} \times\{t\}\right)
$$

and, correspondingly,

$$
\Gamma_{\rho, T}:=\left\{(x, t) \in \mathbb{R}^{n} \times(0, T] ; x \in \Gamma_{\rho(t)}\right\}=\bigcup_{t \in(0, T]}\left(\Gamma_{\rho(t)} \times\{t\}\right) .
$$

Observe that $\Omega_{0, T}$ is just the standard parabolic cylinder $\Omega \times(0, T]$. Similarly, $\Gamma_{0, T}=\Gamma \times(0, T]$. For the sake of completeness, we write $J_{T}:=$ $J \times(0, T]$.

Then, given any initial value $\rho_{0} \in \mathcal{U}$, consider the moving boundary problem of determining a pair $(u, \rho)$ satisfying the following set of equations:

$$
\begin{aligned}
& \Delta u=0 \quad \text { in } \quad \Omega_{\rho, T} \\
& u=\sigma \kappa_{\rho} \quad \text { on } \quad \Gamma_{\rho, T} \\
& (1-\delta) u+\delta\left(\nabla u \mid \nu_{J}\right)=b \quad \text { on } \quad J_{T} \\
& \partial_{t} N_{\rho}-\left(\nabla u \mid \nabla N_{\rho}\right)=0 \quad \text { on } \quad \Gamma_{\rho, T} \\
& \rho(0, \cdot)=\rho_{0} \quad \text { on } \quad \Gamma \text {. }
\end{aligned}
$$

Here, $\sigma>0$ is a positive constant, called surface tension, and $\kappa_{\rho(t)}(x)$ denotes the mean curvature of $\Gamma_{\rho(t)}$ at $x \in \Gamma_{\rho(t)}, t \in[0, T]$. We use the sign convention that convex hypersurfaces have positive mean curvature. In particular, we have $\kappa_{0} \equiv 1$ if $\Gamma$ is the unit sphere. Moreover, $\Delta$ and $\nabla$ stand for the Laplacian and the gradient, respectively, in the Euclidean metric. The outer unit normal field over $J$ is denoted by $\nu_{J}$ and $N_{\rho}$ is a defining function for $\Gamma_{\rho}$, i.e., $N_{\rho}^{-1}(0)=\Gamma_{\rho}, \rho \in \mathcal{U}$. A precise definition of $N_{\rho}$ is given below. Finally, the function $b$ is given and with the integer $\delta \in\{0,1\}$ we either choose a Dirichlet or a Neumann type boundary condition on the fixed part $J$ of the boundary. The entire system (1.1) is called classical formulation of the one-phase Hele-Shaw model with surface tension. To state our results clearly, we need some notations. Let us first give the definition of $N_{\rho}$. For this pick $a_{0} \in(0, \operatorname{dist}(\Gamma, J))$ and let

$$
\mathcal{N}: \Gamma \times\left(-a_{0}, a_{0}\right) \rightarrow \mathbb{R}^{n}, \quad \mathcal{N}(x, \lambda):=x+\lambda \nu(x) .
$$

Then $\mathcal{N}$ is a smooth diffeomorphism onto its image $\mathcal{R}:=i m(\mathcal{N})$, i.e.,

$$
\mathcal{N} \in \operatorname{Diff}^{\infty}\left(\Gamma \times\left(-a_{0}, a_{0}\right), \mathcal{R}\right),
$$


provided $a_{0}>0$ is small enough. It is convenient to decompose the inverse of $\mathcal{N}$ into $\mathcal{N}^{-1}=(X, \Lambda)$, where

$$
X \in C^{\infty}(\mathcal{R}, \Gamma) \quad \text { and } \quad \Lambda \in C^{\infty}\left(\mathcal{R},\left(-a_{0}, a_{0}\right)\right) .
$$

Given $\rho \in \mathcal{U}$, define now

$$
N_{\rho}: \mathcal{R} \rightarrow \mathbb{R}, \quad N_{\rho}(y):=\Lambda(y)-\rho(X(y)) .
$$

Then it is not difficult to verify that $\Gamma_{\rho}=N_{\rho}^{-1}(0)$.

Next we introduce some function spaces. Given an open subset $U$ of $\mathbb{R}^{n}$, let $h^{s}(U)$ denote the little Hölder space of order $s>0$, i.e., the closure of $B U C^{\infty}(U)$ in $B U C^{s}(U)$, the Banach space of all bounded and uniformly Hölder continuous functions of order $s$. We use the symbol $C^{s}(U)$ to denote the Fréchet space of all locally Hölder continuous functions of order $s$. If $M$ is a (sufficently) smooth submanifold of $\mathbb{R}^{n}$ the spaces $h^{s}(M)$ are defined by means of a smooth atlas for $M$. Moreover, we need the anisotropic function spaces $C^{0,2+\alpha}\left(\Omega_{\rho, T}\right)$ consisting of all $u: \Omega_{\rho, T} \rightarrow \mathbb{R}$ such that, given $(x, t) \in \Omega_{\rho, T}$, the function $u(\cdot, t)$ belongs to $C^{2}\left(\Omega_{\rho(t)}\right) \cap h^{1+\alpha}\left(\Omega_{\rho(t)}\right)$ and the function $u(x, \cdot)$ belongs to $C((0, T])$. Finally, we fix $\beta \in(\alpha, 1)$ and we set

$$
\mathcal{V}:=h^{2+\beta}(\Gamma) \cap \mathcal{U}
$$

A pair $(u, \rho)$ is called a classical Hölder solution of (1.1), if $(u, \rho) \in$ $C^{0,2+\alpha}\left(\Omega_{\rho, T}\right) \times\left(C([0, T], \mathcal{V}) \cap C\left((0, T], h^{3+\alpha}(\Gamma)\right) \cap C^{1}\left((0, T], h^{\alpha}(\Gamma)\right)\right)$ and if $(u, \rho)$ satisfies the equations in (1.1) point-wise. Recall that the function $b$ and the surface tension $\sigma$ are known quantities, i.e., we assume that

$$
b \in h^{1+\alpha-\delta}(J) \quad \text { and } \quad \sigma>0
$$

are given. Our main result for problem (1.1) now reads as follows:

Theorem 1. Given any initial value $\rho_{0} \in \mathcal{V}$, there exists a unique classical Hölder solution $(u, \rho)$ of (1.1) on a sufficiently small interval of existence $(0, T]$. Moreover, the moving boundary $\rho:(0, T) \rightarrow \mathcal{V}$ is analytic in the time variable.

Of course, we can choose $\rho_{0} \equiv 0$ above. Then Theorem 1 guarantees a classical solution to (1.1) starting from the initial hypersurface $\Gamma$, which we picked for simplicity to be $C^{\infty}$. Observe, however, that we also get a classical solution to problem (1.1) for any $C^{2+\beta}$ initial hypersurface $\Gamma_{\rho_{0}}$ which is close to $\Gamma$ in the sense that $\rho_{0}$ belongs to $\mathcal{V}$.

Let us also discuss the above result comparing it to the one-phase problem without surface tension, given by $\sigma=0$. In the latter, the sign of the function $b$ becomes significant. Recently, it was shown in [10] that the one-phase Hele-Shaw problem is well-posed if $b \succeq 0$, i.e., if $b(x) \geq 0$ for $x \in J$ but $b \not \equiv 0$. This is in clear contrast to the case $\sigma>0$ where the sign 
of $b$ has no influence on the problem being well-posed, as Theorem 1 shows. Hence surface tension has a regularizing effect on Hele-Shaw models, see also [7], [14], [13], and [12].

We call problem (1.1) linearly ill-posed on $\mathcal{V}$ if the linearized equation on a fixed reference domain is ill-posed in the sense of Hadamard. Then we have the following sharp alternative:

Theorem 2. The Hele-Shaw problem (1.1) is well-posed on $\mathcal{V}$ if $\sigma>0$ or if $\sigma=0$ and $b \succeq 0$. It is linearly ill-posed on $\mathcal{V}$ if $\sigma<0$ or if $\sigma=0$ and $b \preceq 0$.

Let us now turn to the two-phase Hele-Shaw model. To begin with, assume again that $\Omega^{1}$ is a bounded smooth domain in $\mathbb{R}^{n}$ such that its boundary $\partial \Omega^{1}$ consists of two disjoint components, the interior part $J^{1}$ and the exterior part $\Gamma$. In addition, let also $\Omega$ be a bounded smooth domain in $\mathbb{R}^{n}$ containing $\Omega^{1}$ and possessing a boundary with two disjoint components. The interior part of $\partial \Omega$ is assumed to coincide with $J^{1}$ and the exterior part is called $J^{2}$. Finally, we let $\Omega^{2}:=\Omega \backslash \bar{\Omega}^{1}$ and we use the same notation as above for $\mathcal{U}, \Gamma_{\rho}, \Gamma_{\rho, T}, N_{\rho}, J_{T}^{i}$, and $\Omega_{\rho, T}^{i}, i \in\{1,2\}$ and $T>0$. Of course, in this situation we assume that the positive constant $a$ in $\mathcal{U}$ is chosen small enough so that $\Gamma_{\rho}$ intersects neither $J^{1}$ nor $J^{2}$. Then we consider the following two-phase problem: Given an initial value $\rho_{0} \in \mathcal{V}$, find a triple $\left(u^{1}, u^{2}, \rho\right)$ such that

$$
\begin{aligned}
\left(u_{1}, u_{2}\right) & \in C^{0,2+\alpha}\left(\Omega_{\rho, T}^{1}\right) \times C^{0,2+\alpha}\left(\Omega_{\rho, T}^{2}\right) \\
\rho & \in C([0, T], \mathcal{V}) \cap C\left((0, T], h^{3+\alpha}(\Gamma)\right) \cap C^{1}\left((0, T], h^{\alpha}(\Gamma)\right)
\end{aligned}
$$

and such that

$$
\begin{aligned}
& \Delta u^{i}=0 \quad \text { in } \quad \Omega_{\rho, T}^{i} \\
& \left(\nabla u^{i} \mid \nu_{J}\right)=0 \quad \text { on } \quad J_{T}^{i} \\
& u^{i}=\sigma \kappa_{\rho} \quad \text { on } \quad \Gamma_{\rho, T} \\
& \partial_{t} N_{\rho}-\left(\nabla u^{1}-\nabla u^{2} \mid \nabla N_{\rho}\right)=0 \quad \text { on } \quad \Gamma_{\rho, T} \\
& \rho(0, \cdot)=\rho_{0} \quad \text { on } \quad \Gamma \text {. }
\end{aligned}
$$

Here we use the sign convention that $\kappa_{\rho}$ denotes the mean curvature of $\Gamma_{\rho}$ with respect to $\Omega_{\rho}^{1}$. System (1.3) is the classical formulation of the twophase Hele-Shaw model with surface tension. For problem (1.3) we have the following general existence, uniqueness, and regularity result:

Theorem 3. Assume that $\sigma>0$. Then, given any initial value $\rho_{0} \in$ $\mathcal{V}$, problem (1.3) possesses a unique classical solution $\left(u^{1}, u^{2}, \rho\right)$ in the class (1.2) for a sufficiently small $T>0$. Moreover, the interface depends 
analytically on the time variable. If $\sigma<0$, then problem (1.3) is linearly ill-posed on $\mathcal{V}$.

Consider the one-phase problem (1.1) and suppose we start with an initial domain $\Omega$ such that the mean curvature $\kappa_{0}$ of $\Gamma$ takes positive and negative values. Since the function $\kappa_{0}$ enters in the elliptic problem containted in (1.1), it is impossible to use any elliptic comparison principle for problem (1.1). Consequently, unlike the Hele-Shaw model without surface tension, problem (1.1) is generally not endowed with any monotonicity properties. This explains to some extend the fact that even weak solutions to problem (1.1) were not known to exist in general. In the twodimensional case, X. Chen [4] proved the existence of weak solutions to (1.3) and global existence when the initial curve is nearly circular. Under even more restictive assumptions, i.e., when the initial curves are small analytic perturbations of circles, P. Constantin and M. Pugh [6] established existence of analytic solutions to (1.1). Their approach uses conformal transformations and is therefore strictly restricted to the two-dimensional case.

Our approach to problem (1.1) and to problem (1.3) is of a completely different nature and has the advantage of working in any space dimension and for general initial domains. The proofs of the above results are based on the techniques developed in [8], [9], and [10] and will be published in [11]. Nevertheless, let us briefly explain the procedure for the one-phase Hele-Shaw model (1.1).

In a first step, we transform the original domains $\Omega_{\rho}$ with a moving boundary to the fixed reference domain $\Omega$. The corresponding diffeomorphisms, which will of course depend nonlinearly on the function $\rho$, induce a Riemannian metric $g_{\rho}$ on the reference domain $\Omega$. Let $\Delta_{\rho}$ denote the Laplace-Beltrami operator with respect to $\left(\Omega, g_{\rho}\right)$ and let $\partial_{\nu_{J}}$ and $\partial_{N_{\rho}}$ stand for the directional derivatives with respect to the outer normal unit on $J$ and to $\nabla N_{\rho}$, repectively. Moreover, let $h_{\rho}$ denote the mean curvature of $\left(\Gamma, g_{\rho}\right)$ with respect to $\left(\Omega, g_{\rho}\right)$. The transformed version of problem (1.1) then reads as follows:

$$
\begin{aligned}
& \Delta_{\rho} v=0 \quad \text { in } \quad \Omega_{0, T} \\
& v=\sigma h_{\rho} \quad \text { on } \quad \Gamma_{0, T} \\
& (1-\delta) v+\delta \partial_{\nu_{J}} v=b \quad \text { on } \quad J_{T} \\
& \partial_{t} \rho+\partial_{N_{\rho}} v=0 \quad \text { on } \quad \Gamma_{0, T} \\
& \rho(0, \cdot)=\rho_{0} \quad \text { on } \quad \Gamma \text {. }
\end{aligned}
$$

It is known that the principal part of the mean curvature $h_{\rho}$ is a quasilinear 
second order elliptic operator. This means in particular that there exist

$$
P \in C^{\omega}\left(\mathcal{U}, \mathcal{L}\left(h^{3+\alpha}(\Gamma), h^{1+\alpha}(\Gamma)\right) \quad \text { and } \quad l \in C^{\omega}\left(\mathcal{U}, h^{1+\alpha}(\Gamma)\right)\right.
$$

such that

$$
h_{\rho}=P(\rho) \rho+l(\rho) \quad \text { for } \quad \rho \in h^{3+\alpha}(\Gamma) \cap \mathcal{U} .
$$

Given $\rho \in \mathcal{U}$ and $r \in h^{1+\alpha}(\Gamma)$, it follows from Theorem 4.3.4 in [16] that the elliptic boundary value problem

$$
\Delta_{\rho} v=0 \quad \text { in } \quad \Omega, \quad v=r \quad \text { on } \quad \Gamma, \quad(1-\delta) v+\partial_{\nu_{J}} v=b \quad \text { on } \quad J
$$

possesses a unique solution in $C^{2}(\Omega) \cap h^{1+\alpha}(\Omega)$. For simplicity we assume that $b=0$ and we call the solution $v_{\rho}(r)$. Then we define

$$
\Phi(\rho) \tau:=\sigma \partial_{N_{\rho}}\left(v_{\rho}(P(\rho) \tau)\right) \quad \text { and } \quad F(\rho):=-\sigma \partial_{N_{\rho}}\left(v_{\rho}(l(\rho))\right)
$$

for $\rho \in \mathcal{U}$ and $\tau \in h^{3+\alpha}(\Gamma)$. Using the definition of $v_{\rho}(\cdot)$ and the equations in (1.5), it is not difficult to see that the following abstract quasilinear evolution equation

$$
\partial_{t} \rho+\Phi(\rho) \rho=F(\rho), \quad \rho(0)=\rho_{0},
$$

is equivalent to problem (1.4) and therefore also to problem (1.1), being a transformed version of (1.4). A careful analysis of the operator $\Phi$ discloses that $[\rho \mapsto \Phi(\rho) \rho]$ is a nonlinear pseudo-differential operator of third order. In addition, the operator $\Phi$ carries a quasilinear structure, i.e.,

$$
\Phi \in C^{\omega}\left(\mathcal{U}, \mathcal{L}\left(h^{3+\alpha}(\Gamma), h^{\alpha}(\Gamma)\right)\right) .
$$

Moreover, it turns out that $F$ is a second order term in the sense that

$$
F \in C^{\omega}\left(\mathcal{U}, h^{\alpha}(\Gamma)\right) .
$$

We shall use the theory of abstract quasilinear parabolic problems developed by H. Amann [2] to investigate problem (1.6). See also the results in [3] and [15]. A thorough knowledge of the corresponding linear equation is fundamental in order to apply this theory. Indeed, using Fourier multiplier representations of Poisson operators and subtle perturbation arguments it can be verified that the operator $-\Phi(\rho)$ generates for each $\rho \in \mathcal{V}$ a strongly continuous analytic semigroup on the space $h^{\alpha}(\Gamma)$, cf. Section 5 in [11]. Once this generation property is verified, a unique classical solution of problem (1.6) is guaranteed by Theorem 12.1 in [2], see also Theorem 2.11 in [3] and Theorem 3.1 in [15]. 


\section{Acknowledgment}

We would like to thank Sigurd Angenent for helpful suggestions leading to an improvement of preliminary results.

\section{Note added in proof}

After this paper was submitted, we learned of a manuscript by X. Chen, J. Hong, and F. Yi [5] in which also existence and uniqueness of classical solutions for the two-phase problem (1.4) is proved. However, it is noteworthy to mention that Chen et al. assume that the initial hypersurface belongs to $\mathcal{V} \cap C^{3+\alpha}(\Gamma)$ which is a true subspace of $\mathcal{V}$.

\section{References}

1. N. Alikakos, P. Bates, and X. Chen, Convergence of the Cahn-Hilliard Equation to the Hele-Shaw model, Arch. Rational Mech. Anal. 128 (1994) 164-205.

2. H. Amann, Nonhomogeneous linear and quasilinear elliptic and parabolic boundary value problems, in H. J. Schmeisser, H. Triebel, editors, Function Spaces, Differential Operators and Nonlinear Analysis, Teubner, Stuttgart, Leipzig, 1993, 9-126.

3. S. B. Angenent, Nonlinear analytic semiflows, Proc. Roy. Soc. Edinburgh 115A (1990), 91-107.

4. X. Chen, The Hele-Shaw problem and area-preserving curve shortening motion, Arch. Rational Mech. Anal. 123 (1993), 117-151.

5. X. Chen, J. Hong, and F. Yi, Existence, uniqueness, and regularity of classical solutions of the Mullins-Sekerka problem, Preprint (1996).

6. P. Constantin and M. Pugh, Global solutions for small data to the Hele-Shaw problem, Nonlinearity 6 (1993), 393-415.

7. C. M. Elliott and J. R. Ockendon, Weak and Variational Methods for Moving Boundary Problems, Pitman, Boston, 1982.

8. J. Escher and G. Simonett, Maximal regularity for a free boundary problem, Nonl. Diff. Equs. Appl. 2 (1995), 467-510.

9. __ Analyticity of the interface in a free boundary problem, Math. Ann., to appear.

10. __ Classical solutions of multi-dimensional Hele-Shaw models, SIAM J. Math. Anal., to appear.

11. __ Classical solutions for Hele-Shaw models with surface tension, Preprint (1996).

12. M. E. Gurtin, Thermomechanics of Evolving Phase Boundaries in the plane, Clarendon Press, Oxford, 1993. 
13. S. D. Howinson, Complex variable methods in Hele-Shaw moving boundary problems, European J. Appl. Math. 3 (1992), 209-224.

14. A. A. Lacely, S. D. Howinson, and J. R. Ockendon, Irregular morphologies in unstable Hele-Shaw free boundary problems, Quart. J. Mech. Appl. Math. 43 (1990), 387-405.

15. G. Simonett, Quasilinear parabolic equations and semiflows. In Evolution Equations, Control Theory, and Biomathematics, Lecture Notes in Pure and Appl. Math., M. Dekker, New York, 155, (1994) 523-536.

16. H. Triebel, Theory of Function Spaces, Birkhäuser, Basel, 1983.

Mathematical Institute, University of Basel, CH-4051 Basel, Switzerland

E-mail address: escher@math.unibas.ch

Department of Mathematics, Vanderbilt University, Nashville, TN 37240 , U.S.A.

E-mail address: simonett@math.vanderbilt.edu 